\title{
Route Choice Research Based on Fast and Hierarchical Mobile Multicast
}

\author{
Zuhong Feng, Bin Li \\ School of Computer Science and Engineering, Beifang University of Nationalities \\ Yinchuan, China \\ E-mail: seek_li@aliyun.com
}

\begin{abstract}
Because of the high scalability and efficiency of multicast, more and more people pay attention to mobile multicast. The fast and hierarchical mobile multicast is one of the excellent multicast algorithms, but the transmission path in the fast and hierarchical mobile multicast is not the best. This paper through adds the mobile device's staying time to solve this problem. When a mobile device need to move to a new access router, the mobile device prediction the staying time in new router at first, then the new router according to the staying time to choose a suitable path joins the multicast group. Empirical results show that this new algorithm can optimize routing path and save network resources.
\end{abstract}

Keywords—mobile; multicast; hierarchical mobile; prediction; optimize

\section{INTRODUCTION}

About mobile multicast, IETF has proposed two basic algorithms to support mobile multicast $[1,2]$. They are remote subscription and home subscription. In home subscription, home agent replaces mobile device joins/leaves the multicast group. Then a bi-directional tunnel will be constructed between the mobile device and its home agent, and mobile device receives/sends packets through this tunnel. But when the mobile device is far from its home agent, the tunnel will be very long. On this condition, it will waste network resources. In remote subscription, mobile device sends a join request message to the multicast router on the new network, and mobile device joins the multicast group directly via this router. So in remote subscription the transmission path is optimization, the load of the network system is minimal, and little modification of existed protocols is needed. But whenever the mobile device moves to a new router it will rejoin the multicast group, which will cause the multicast delivery tree to rebuild frequently and the Service Disruption Time is quite long [3].

In view of the above-mentioned facts, people proposed a series of modified approaches. Hierarchical mobile multicast architecture uses a hierarchical architecture to isolate the local movement from outside. When mobile devices move under local movement, binding messages just update in local, so it can improve the stability of the multicast delivery tree [4]. Furthermore, introduce the fast handoff mechanism can reduce handoff delay and packets loss rate for inter-domain handoff and intra-domain handoff [5]. Fast handoff and hierarchical mobile multicast architecture occupies a very important position in mobile multicast [6]. However, in fast handoff and hierarchical mobile multicast, when the location of mobile node changes frequently, the transmission path isn't optimization. Then this paper will analyze this problem and solve it.

\section{THE OPTIMIZATION OF MULTICAST TREE}

There are two basic router algorithms in multicast.

1) Shortest path tree algorithm: Finds the path with the lowest cost between the source node and every other node in the multicast group.

2)Minimal spanning tree algorithm: Connect all nodes in the multicast group, and the cost of all links is the lowest.

When a node joins a multicast group, this node can choose either of the above-algorithms, as showed in "Fig. 1".

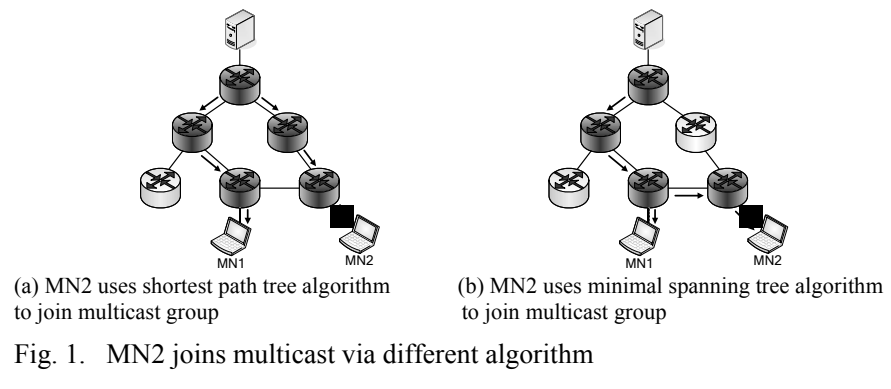

However, different way to join a multicast group will impact on the stability of the multicast delivery tree.

As showed in "Fig. 2" left, MN1 leaves the multicast group earlier than $\mathrm{MN} 2$, at right $\mathrm{MN} 2$ leaves the multicast group earlier than MN1. If MN2 uses the shortest path tree algorithm to join the multicast group, after MN1 left, the multicast delivery tree prunes MN1's link. This moment MN2 still staying at the multicast delivery tree and the transmission path is optimization.

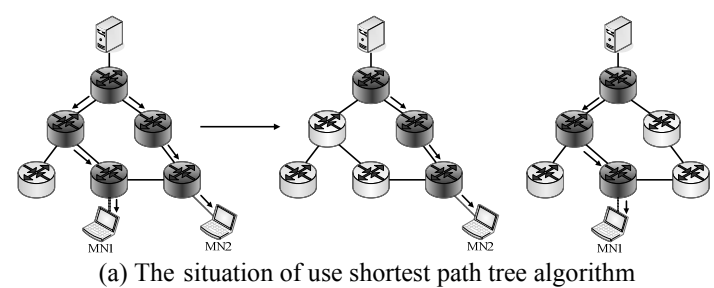

(a) The situation of use shortest path tree algorithm 


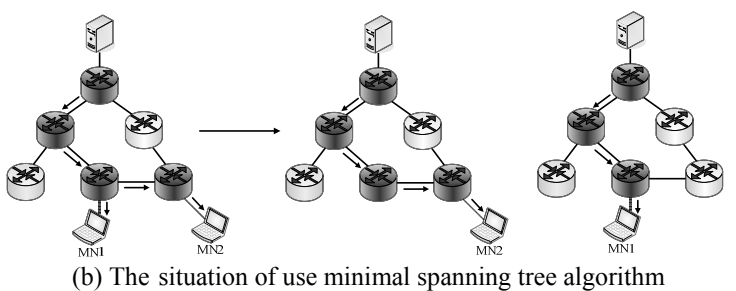

Fig. 2. The update of multicast delivery tree about use two different ways to join the multicast group

When MN2 uses minimal spanning tree algorithm to join and MN1 leaves the multicast group earlier than MN2. After MN1 left, because of MN2 still needs through MN1's link to receive the multicast packets, so multicast delivery tree can't prune MN1's link. This kind of situation will waste network resources.

If MN2 uses shortest path tree algorithm to join the multicast group, and MN2 leaves the multicast group earlier than MN1. After MN2 left, the multicast delivery tree prunes MN2's link, MN1 will get optimization routing path. When MN2 uses minimal spanning tree algorithm to join the multicast group, and MN2 leaves the multicast group earlier than MN1. After MN2 left, the multicast delivery tree prunes MN2's link, MN1 still get optimal routing path.

However, the path when MN2 uses minimal spanning tree algorithm to join the multicast group will be shorter than uses the shortest path tree algorithm to join the multicast group. So If MN2 uses minimal spanning tree algorithm to join the multicast group, there is less update for multicast delivery tree. In conclusion, If MN2 leaves the multicast group earlier than MN1, MN2 uses minimal spanning tree algorithm to join the multicast group can obtain better routing path. Else MN2 uses shortest path tree algorithm can obtain better routing path.

\section{OPTIMIZED ALGORITHMS}

Add mobility prediction can optimize routing path and save network resources. In the new method, when mobile node finds a New Access Point (NAP), mobile node will predict its residence time under the NAP. If it's staying time is long, the mobile node will use shortest path tree algorithm to join the multicast group, else in order to save network resources, the mobile node will use minimal spanning tree algorithm to join the multicast group.

\section{A. Shortest path tree algorithm}

Shortest path tree algorithm requires the distance from multicast access point to every other node is the shortest. Multicast access point will be timed to broadcast messages to all nodes in the network. In these messages, there is a parameter show the distance from multicast access point to other nodes. Initially the value of the distance parameter is 1 , when the next hop receives these messages, the value of distance parameter will plus one, and then continues to send these messages to its next hop. So, when nodes receive these messages, it will know the distance from multicast access point to it. If nodes receive these messages more than one at the same time, the smallest distance parameter is the distance from multicast access point to it.

\section{B. Minimal spanning tree algorithm}

Minimal spanning tree algorithm requires the distance of all links is the shortest. If a node uses minimal spanning tree algorithm to join a multicast group, first, the node broadcasts a message to all nodes, when nodes on the multicast tree receive this message, they will respond a message, and this message also has a distance parameter. Initially the value of the distance parameter is 1 , when the next hop receives this message, the value of distance parameter will plus one. Thanks to the distance parameter, when the node who wants to join the multicast group receives this message, it can know the distance from the multicast tree to it. If the node receives these messages more than one at the same time, the smallest distance parameter is the distance from the multicast tree to it.

\section{Mobility Prediction}

As a result of the heterogeneity of network, the coverage of different Access Point (AP) may be not identical. It makes a considerable influence on staying time [7]. Utilize "Average Staying Time" to prediction staying time can have a satisfactory result. Average Staying Time of an AP is the ratio of stay time and his covering radius.

Considering the movement of a mobile node is random, but also has a certain historical continuity, so we can predict the average speed in the future, base on the present Average Staying Time and the historical Average Staying Time.

Exponential Smoothing is a common algorithm [8]; its advantage is simple and less needs to maintain information. In Adaptive Response Rate Single Exponential Smoothing (ARRSES) there is a dynamic parameters named $\alpha$, according to the error between prediction value and practical value, the value of $\alpha$ can make adjustments. Here is the way to compute the Average Staying Time under NAP, as in (1).

$$
\hat{X}_{i+1}=\alpha_{i} X_{i}+\left(1-\alpha_{i}\right) \hat{X}_{i}
$$

$\hat{X}_{i+1}$ and $\hat{X}_{i}$ is the predicted value of Average Staying Time, while mobile node under the (i+1)th AP and the ith AP; $X_{i}$ is the practical value of Average Staying Time, while mobile node under the ith AP; $\alpha_{\mathrm{i}}$ is smoothing parameter, and $0 \leq \alpha_{\mathrm{i}} \leq 1$. Before calculating prediction value of Average Staying Time, need to recalculate the value of $\alpha_{i}$. Here are the steps to calculate $\alpha_{\mathrm{i}}$ :

$$
\begin{gathered}
e_{i}=X_{i}-\hat{X}_{i} \\
E_{i}=\beta e_{i}+(1-\beta) E_{i-1} \\
M_{i}=\beta\left|e_{i}\right|+(1-\beta) M_{i-1} \\
\alpha_{i}=\left|E_{i} / M_{i}\right|
\end{gathered}
$$

The $e_{i}$ is prediction error of the ith AP; $\beta$ is a constant, usually the value of $\beta$ is 0.1 or $0.2 ; E_{i}$ is the prediction value of smoothing error. If the value of $\alpha_{i}$ near to 0 , it shows that the prediction can be controlled. If the value of $\alpha_{i}$ near to 1 , it shows that the prediction value is biased. If we substitute $\alpha_{i}$ 
into (1), we can work out $\hat{X}_{i+1}$. Then according to (6), we can work out the Staying Time of NAP.

$$
\hat{T}_{i+1}=\hat{X}_{i+1} \times R_{i+1}
$$

\section{Analysis}

Out of the mobile nodes in the network, the location of other nodes almost no change, and when the network calculates the distance, there is no relationship to the mobile nodes. Therefore, the path of shortest path tree with no need for frequent update, and the update of the minimal spanning tree just when nodes use minimal spanning tree algorithm to join the multicast group will happen, so the messages exchange is lower. The calculation of forecast is happening in the prehandover time, so the forecast algorithm almost no impact on delay time.

\section{SIMULATION}

In this section, we use NS2 to simulate the new algorithm. Firstly we use $\mathrm{C}++$ language to modify the underlying code, add distance parameter to message, and make the routers can receive an identifier. This identifier will show the routers use which path to join the multicast group. Then change the tcl file, let it can use the new algorithm to join a multicast group.

In order to verify this routing algorithm, we set up a symmetric network topology by using tcl language, as showed in "Fig. 3", and set the bandwidths and time delays of all links in network topology are the same. Now we don't consider the mobile node temporarily. At first node 3 joins the multicast group, then node 5 uses minimal spanning tree algorithm to join the multicast group. As a result, node 5 receives the multicast packets via node 3 , but not node 4 .

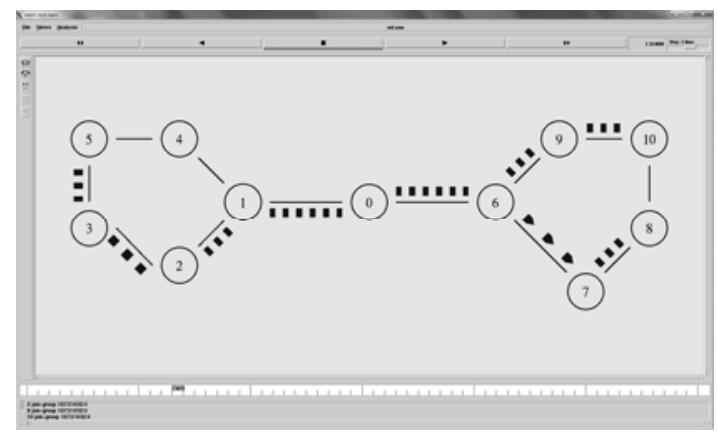

Fig. 3. Simulation of two nodes use different way to join multicast group
At right, node 8 joins the multicast group earlier than node 10 , then node 10 uses shortest path tree algorithm to join the multicast group. As saw in Figure 3, node 10 receives multicast packets via node 9 , but not the nearly node-node 8 .

\section{CONCLUSION}

About existing mobile multicast routing algorithm, this paper had analyzed the problems of path choice at first, based on these problems we had introduced a new method to optimize the transmission path. Through the simulation on NS2, we had shown that the algorithm is feasible. By analyzing this algorithm, the result display that this algorithm can optimization the cost of update multicast tree, transport delay, packet loss rate and so on, it can effectively save the network resources.

\section{REFERENCES}

[1] Johnson, David, C. Perkins, and Jari Arkko. "RFC 3775: Mobility support in IPv6." IETF, June (2004).

[2] Arkko, Jari, Charles Perkins, and Dave Johnson. "Mobility support in IPv6." (2011).

[3] Romdhani, Imed, et al. "IP mobile multicast: Challenges and solutions." Communications Surveys \& Tutorials, IEEE 6.1 (2004): 18-41.

[4] Soliman, Hesham, Ludovic Bellier, and Karim El Malki. "Hierarchical mobile IPv6 mobility management (HMIPv6)." (2005).

[5] Jung, Hee Young, et al. "Fast handover for hierarchical MIPv6 (FHMIPv6)." IETF draft (2005).

[6] Koodli, Rajeev. "Fast handovers for mobile IPv6." (2005).

[7] Suh, Jin Park and Young-Joo. "A timer-based mobile multicast routing protocol in mobile networks." Computer Communications 17(2003):19651974.

[8] Risteski, Dimce, Andrea Kulakov, and Danco Davcev. "Single exponential smoothing method and neural network in one method for time series prediction." Cybernetics and Intelligent Systems, 2004 IEEE Conference on. Vol. 2. IEEE, 2004. 